\title{
Effects of Hybrid Flow Control on a Normal Shock Boundary-Layer Interaction
}

\author{
Stefanie M. Hirt ${ }^{1}$ and Manan A. Vyas ${ }^{2}$ \\ NASA Glenn Research Center, Cleveland, $\mathrm{OH}, 44135$
}

\begin{abstract}
Hybrid flow control, a combination of micro-ramps and steady micro-jets, was experimentally investigated in the $15 \times 15 \mathrm{~cm}$ Supersonic Wind Tunnel at the NASA Glenn Research Center. A central composite design of experiments method, was used to develop response surfaces for boundary-layer thickness and reversed-flow thickness, with factor variables of inter-ramp spacing, ramp height and chord length, and flow injection ratio. Boundary-layer measurements and wall static pressure data were used to understand flow separation characteristics. A limited number of profiles were measured in the corners of the tunnel to aid in understanding the three-dimensional characteristics of the flowfield.
\end{abstract}

\section{Nomenclature}

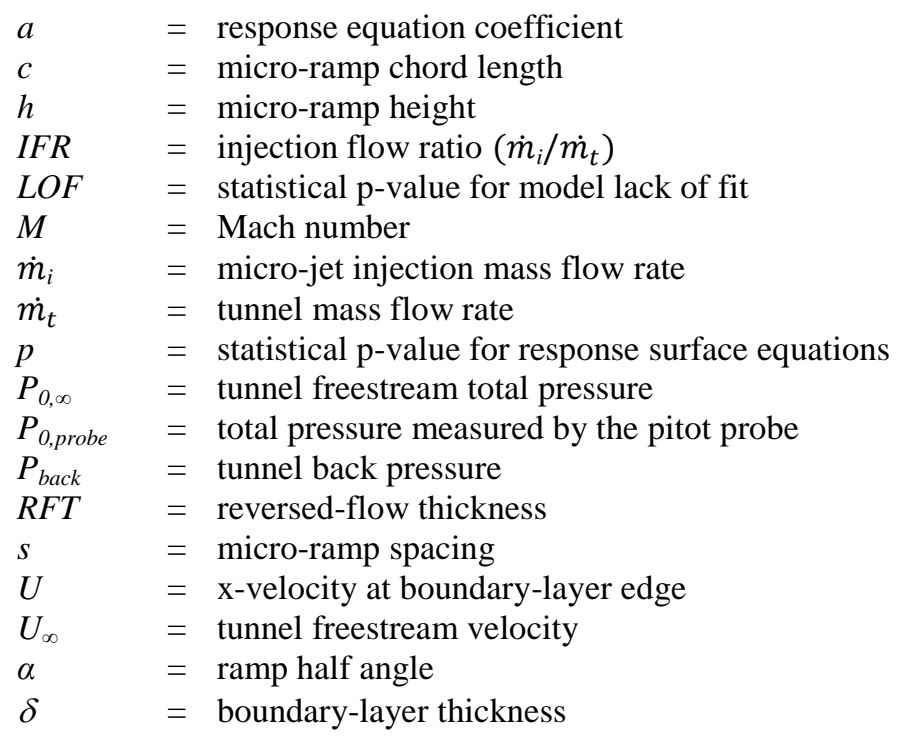

\section{Introduction}

$\mathrm{I}^{\mathrm{n}}$ recent years we have seen an increase in aircraft that feature highly integrated inlets to take advantage of fuelefficient operations, reduced emissions of greenhouse gases, and noise reduction that integrated inlets provide. Engines are embedded within the aircraft fuselage, which reduces the aircraft profile and resulting drag. This reduces fuel consumption and lowers emissions for commercial aircraft. Locating the engines in the fuselage also reduces the heat and noise signature for stealth benefits on military aircraft. However, the advantages come with penalties in the form of separation due to the increased flow-turning angle and shockwave boundary-layer interactions (SBLIs). Such separation causes reduced inlet mass capture, reduced total pressure recovery, and nonuniform flow that contribute to an overall loss of engine performance.

Historically, boundary-layer bleed has been employed for shock-induced boundary-layer separation with traditional vortex generators in the subsonic diffuser; however, for reasons described in Vyas et al. ${ }^{1}$ an alternative

\footnotetext{
${ }^{1}$ Aerospace Engineer, Inlet and Nozzle Branch, MS 5-12. Senior Member AIAA.

${ }^{2}$ Aerospace Engineer, Inlet and Nozzle Branch, MS 5-12. Member AIAA.
} 
means of flow control is studied. Hybrid flow control is a robust approach for pre-conditioning the inlet mass capture and addressing flow separation. Thus, the current work investigates SBLI with hybrid flow control on a contoured surface (bump) to emulate a highly integrated inlet. Hybrid flow control is a combination of micro-ramp ${ }^{2}$ vortex generators (VGs) and steady micro-jets used to energize the boundary layer by transferring and inducing high-momentum flow respectively. VGs have been successfully used in the past for subsonic diffusers to avoid flow separation. Traditional VGs are typically the height of the boundary-layer thickness; however, micro-ramp heights are on the order of $25 \%-40 \%$ of the boundary-layer thickness. Micro-ramps generate a pair of primary counterrotating vortices that transfer the high-momentum flow from the outer boundary layer to the flow near the wall. The mixing due to the primary vortex pair increases the near wall velocity while creating a deficit region in the outer boundary layer and core flow. To reduce this deficit, hybrid flow control introduces a small amount of flow —on the order of $0.25 \%$ of the inlet mass capture per micro-jet - which is injected over the micro-ramps. Pairs of secondary vortices along the ramp centerline and a horseshoe vortex wrapping around are also generated by the ramp. ${ }^{3,4}$ Figure 1 shows the flow features on one-half of a micro-ramp.



Figure 1. Micro-ramp flow features schematic.

An experiment was conducted in the $15 \times 15 \mathrm{~cm}$ Supersonic Wind Tunnel (SWT) at the NASA Glenn Research Center to evaluate the effectiveness of the hybrid flow control for controlling normal shock induced separated flow. Previous efforts ${ }^{5,6,7}$ have studied only micro-ramps as the flow control device. In this effort micro-ramps were tested alone and in the hybrid configuration with steady micro-jets. To emulate an increased flow-turning angle, common in the design of integrated inlets, a bump was used. A total of 17 hybrid flow control configurations were tested during Phase $\mathrm{I}^{1}$ based on a full factorial design of experiments (DOE) method. The variables of interest were interramp spacing $(s)$, micro-ramp height $(h)$, mirco-ramp chord length $(c)$, and micro-jet injection flow ratio $(I F R)$. Posttest DOE analysis showed that the full factorial design was insufficient to obtain an accurate response surface equation. The experimental data had significant curvature, which means second-order effects, and two- and threelevel interaction terms were needed for the prediction model. To capture these effects a new central composite design was used and a test matrix was developed for the Phase II experimental study. The new test matrix was an expansion of the Phase I test matrix so that the cases studied during Phase I were mapped into the Phase II test matrix to avoid unnecessary repeats. This matrix resulted in 8 additional hybrid flow control configurations being tested to complete the new central composite DOE. In order to confirm repeatability the key cases, including the baseline case, were replicated during Phase II.

This paper provides a summary of the Phase I and Phase II experimental results. Details of the flowfield for the baseline and injection only configurations are discussed. Then details of the DOE analysis based on the central composite design and the resulting response surface equations are presented for boundary-layer thickness and reversed-flow thickness. The effects of the factor variables on each response are considered based on plots of the response sureface equation and are supported by examining velocity contours for selected configurations from the DOE. Additionally, an optimization is presented for the current tunnel geometry. 


\section{Experimental Setup}

\section{A. $15 \times 15$ cm Supersonic Wind Tunnel (SWT)}

The $15 \times 15 \mathrm{~cm}$ SWT, shown in Fig. 2, is a continuous flow facility in the Engine Research Building at NASA Glenn Research Center. The inflow is connected to the central air supply, which provides up to $280 \mathrm{kPa}$ (40 psig) pressure at ambient temperature. The supply air is controlled by a valve that adjusts the test section total pressure. The exhaust is captured by the central altitude exhaust system, which is capable of sustaining pressures less than 14 $\mathrm{kPa}(2.0 \mathrm{psia})$.

The tunnel has an exchangeable nozzle capability that allows a speed range for the tunnel from subsonic flow up to Mach 3.0. For the purpose of this experiment, the subsonic (constant area) nozzle block was used to obtain Mach 0.67 flow at the entrance to the test section.

The test section, shown in Fig. 3, has a constant cross-sectional area of $15 \times 15 \mathrm{~cm}(5.91 \times 5.91$ in) and configurable insert sections on the side and bottom walls. For this test the top wall of the test section was comprised of a parabolic bump which created a throat with an area equal to $86.7 \%$ of the non-constricted test section area, as shown in Fig. 3. Immediately after the test section, the flow encounters a rearward facing step where the tunnel area expands to $23.5 \times 23.5 \mathrm{~cm}(9.25 \times 9.25 \mathrm{in})$. To position the normal shock in the test section, a valve downstream of the testsection controlled the back-pressure. The inlet total pressure was set to obtain a constant freestream Reynolds number of $13.1 \mathrm{E} 6 / \mathrm{m}(4.00 \mathrm{E} 6 / \mathrm{ft})$. At these conditions, the tunnel mass flow rate was $4.72 \mathrm{~kg} / \mathrm{s}(10.4 \mathrm{lbm} / \mathrm{s})$.

Figure 3 also shows a schlieren image inset at the appropriate position in the tunnel layout. The normal shock can be seen positioned on the bump, as can a region of separated flow originating at the foot of the normal shock.



Figure 2. 15x15 cm Supersonic Wind Tunnel.



Figure 3. Layout of the tunnel test section with key axial locations. 


\section{B. Micro-ramps and Flow Injection Design}

The micro-ramps were intended to pre-condition the flow and reduce the shock-induced separation on the aft end of the bump. For this purpose, four micro-ramps were positioned laterally in the tunnel, symmetric about the centerline of the test section. Figure 4 shows the micro-ramp geometry in terms of inter-ramp spacing $(s)$, microramp height $(h)$, and micro-ramp chord length $(c)$. These parameters, along with the flow injection rate, were varied in the test matrix. The micro-ramp half angle, $\alpha$, was held constant at 24 degrees, and the axial locations of the micro-jet injection ports and the micro-ramps was also constant at $\mathrm{x}=14.9 \mathrm{~cm}$ and $\mathrm{x}=15.9 \mathrm{~cm}$ respectively, as seen in Fig. 3. Based on previous experience ${ }^{3}$ and a preliminary computational screening study of the variables for this configuration, inter-ramp spacing $(s)$ was investigated at three levels: 25, 30, and $35 \mathrm{~mm}$; ramp height $(h)$ at three levels: 3,4 , and $5 \mathrm{~mm}$; ramp chord length $(c)$ at three levels: 12,18 , and $24 \mathrm{~mm}$; and injection flow ratio (IFR) at three levels: $0.0,0.5$, and $1.0 \%$ of the total tunnel mass flow rate. The injection flow ratio reported is the total for the four injection ports combined.

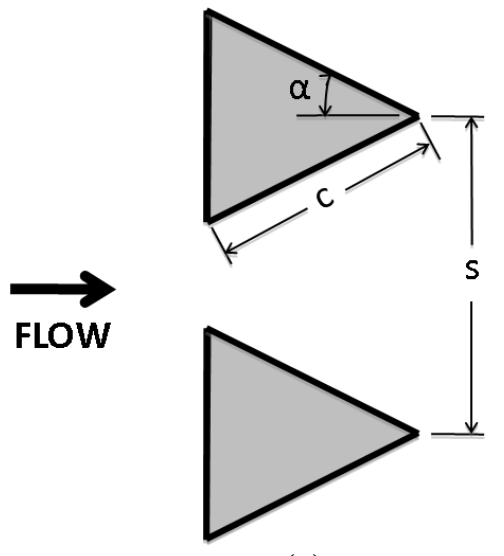

(a)

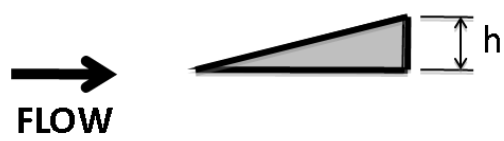

(b)

Figure 4. Micro-ramp geometry parameters shown in (a) Top view and (b) Side view.

Figure 5 shows the 11 micro-ramp configurations (and 1 blank) required for the central composite DOE method where $s, h$, and $c$ were varied. The micro-ramp inserts were mounted on the injection assembly (Fig. 6) which was laterally centered in the tunnel.

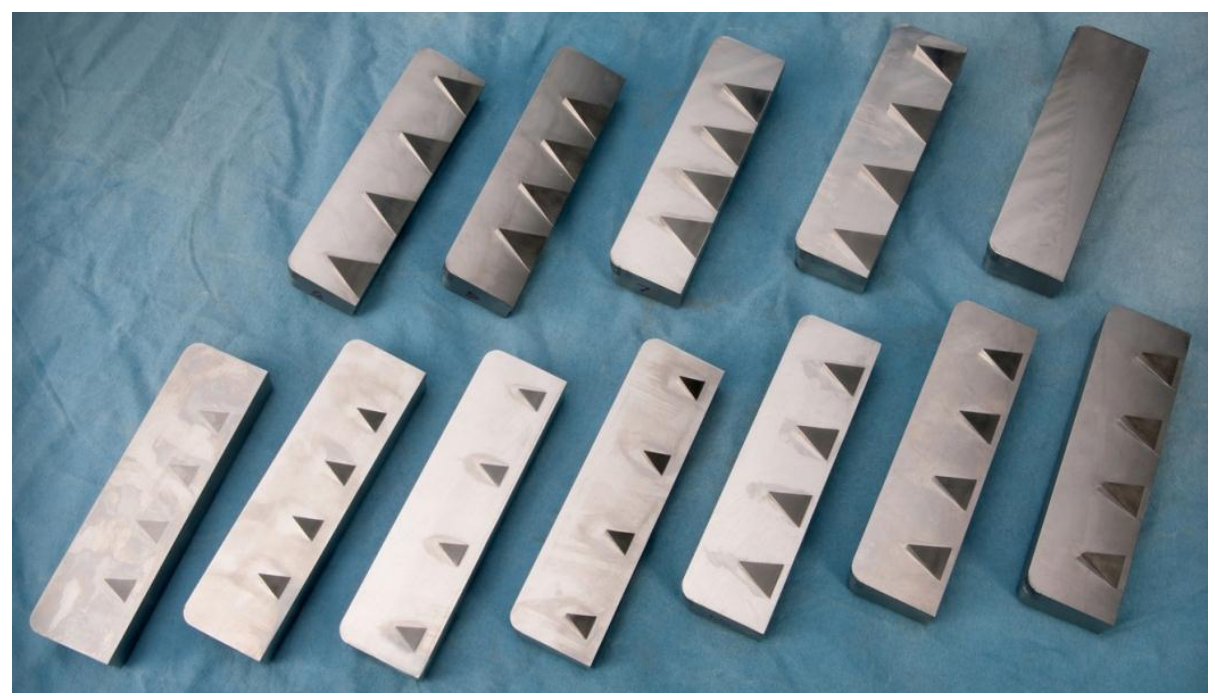

Figure 5. Micro-ramp inserts. 


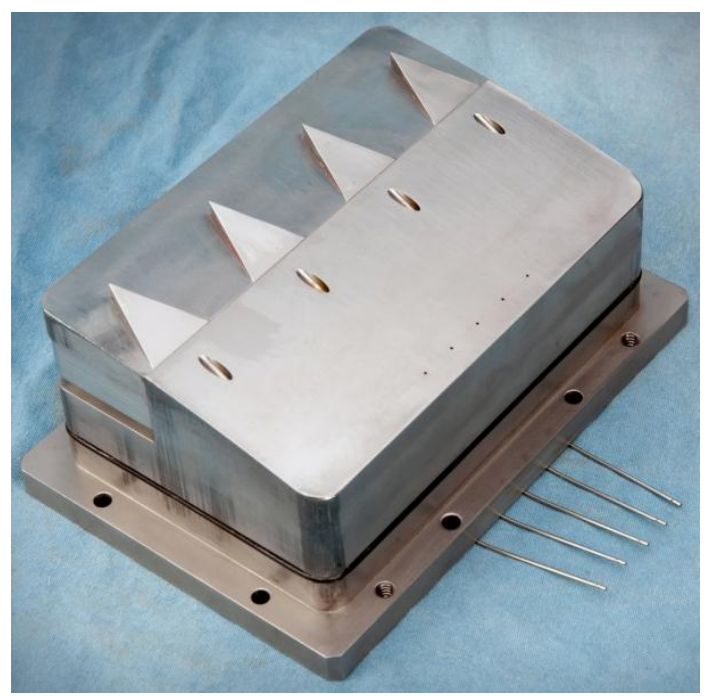

Figure 6. Micro-ramps and injection assembly.

The micro-jet flow injection was intended to reduce the momentum deficit created by the pair of counterrotating vortices which transfers the high-momentum flow from the boundary-layer edge to near the wall. A pre-test computational fluid dynamic (CFD) analysis of $20^{\circ}, 40^{\circ}$, and $60^{\circ}$ injection port angles had shown that the $20^{\circ}$ injection port angle had the most effect on the boundary-layer improvement. Thus, $20^{\circ}$ injection ports were used for this test. Figure 6 shows the micro-ramps and injection assembly, where the injection ports are just upstream of the micro-ramps. The ports were aligned with the micro-ramps so that the flow injection was centered over the microramps. Injection port configurations were fabricated to match each of the three inter-ramp spacings of 25, 30, and 35 $\mathrm{mm}$. The injection air was supplied by a 120 psia supply line. An upstream valve in the line was used regulate the pressure in the injection plenum to maintain the desired micro-jet injection flow ratio.

\section{Instrumentation and Data Systems}

A pitot probe on a translation mechanism was used to measure boundary-layer profiles in the $y-z$ plane at $x \approx 34$ $\mathrm{cm}$. This station is approximately $1 \mathrm{~cm}$ aft of the end of the bump and was in a highly separated region of the flow. The measurement grid is shown in Fig. 7, where each intersection represents a measurement location. For Phases I and II, measurements were collected at z-planes between $-s / 2$ and $+s / 2$. The $10 \mathrm{y}$-profiles were distributed such that there were 6 evenly spaced profiles on the positive-z side, 3 evenly spaced profiles on the negative- $z$ side, and one at $\mathrm{z}=0 \mathrm{~cm}$. This is the region represented in red in the figure. In Phase II, y-profiles were added at $\mathrm{z}= \pm 3.5 \mathrm{~cm}$. For select configurations, corner profiles were completed at a 45-degree angle. Static pressure taps were located in the measurement plane at locations corresponding to the z-locations of the boundary-layer profiles for $\mathrm{s}=30 \mathrm{~mm}$ to allow velocity to be computed. A row of static pressures were measured axially along the centerline of the bump.

Both the tunnel and injection mass flow rates were measured by venturis.



Figure 7. Measurement plane with intersections representing measurement points. Shown for $s=30 \mathrm{~mm}$. 


\section{Design of Experiments}

The outcome of a Design of Experiments (DOE) analysis is a predicted response surface. For this experiment the response variables chosen were the boundary-layer thickness and the reversed-flow thickness. The predicted response surface is defined by an equation that describes how the response variable changes as a function of the factor variables (in this case, $s, h, c$, and $I F R$ ) within the design space. The response surface equation can be used to estimate the value of the response variables at configurations that were not tested.

The boundary-layer thickness, $\delta$, was selected as a response variable because it provides information on the extent to which the flow control impacts the core flow. The boundary-layer thickness was chosen as the y-location where the local velocity reaches $99 \%$ of the freestream velocity. This value was calculated for the boundary-layer profile at each z-location. Then, the values for z-locations from $+s / 2$ to $-s / 2$ were span-averaged on an area weighted basis. This span-averaged value was the response recorded for the DOE analysis.

The span-averaged reversed-flow thickness, RFT, was chosen because it indicates the magnitude of the separation. The reversed-flow thickness was chosen as the largest y-location in a boundary-layer profile with a measured total pressure less than or equal to the static pressure. This value was determined for each boundary-layer profile and span-averaged in the same manner as the boundary-layer thickness.

The incompressible shape factor was considered as a response variable for consistency with previous tests ${ }^{3,8}$, but it could not be calculated for the reversed-flow region because the pitot tube used to measure the boundary-layer profiles could not be used to determine the velocity in the reversed-flow region.

The values of the factor variable that were held constant were determined through a DOE screening study. The purpose of the screening study was to determine the best size, geometry, location and orientation of the hybrid flow control actuators and to set the ranges of the factor variables which should be studied in the DOE itself. In the screening DOE study, there were 5 independent factor (design) variables considered. Using a Main Effects DOE strategy, there were 6 CFD cases run in the screening process. The results of the screening study led to the selection of four factor variables to be included in the current effort and provided the best overall ranges for the selected factor variables. Table 1 specifies the ranges of the four factor variables that were tested.

Table 1: Factor variable ranges

\begin{tabular}{|c|c|c|c|c|}
\hline $\begin{array}{c}\text { Variable } \\
\text { Name }\end{array}$ & Units & -1 level & 0 level & +1 level \\
\hline$s$ & $\mathrm{~mm}$ & 25 & 30 & 35 \\
\hline$h$ & $\mathrm{~mm}$ & 3 & 4 & 5 \\
\hline$c$ & $\mathrm{~mm}$ & 12 & 18 & 24 \\
\hline$I F R$ & $\%$ & 0.0 & 0.5 & 1.0 \\
\hline
\end{tabular}

To test every combination of the four factor variables at the three selected levels, 81 configurations would be needed. In Phase I, a full factorial DOE matrix consisting of 17 configurations was tested. Those configurations represented the corners of the design space as well as the center point to test for curvature. Additionally a baseline configuration with no flow control was tested for comparison. Analysis of the Phase I results showed that a full factorial design was not sufficient to generate a response surface for reversed-flow thickness.

In Phase II, DOE techniques were used to select 8 additional cases, expanding the study to the 25 cases comprising a face-centered central composite design, which provides the first- and second-order effects of each variable and the first-order interactions. The combinations of factor variables for each test point in the central composite design are shown in Table 2 with the configurations tested in Phase I shaded gray. A response equation for boundary-layer thickness is of the form:

$$
\begin{array}{r}
\delta=a_{o}+a_{1} s+a_{2} h+a_{3} c+a_{4} I F R+a_{12} s h+a_{13} s c+a_{14} s I F R+a_{23} h c+a_{24} h I F R+a_{34} c I F R+ \\
a_{11} s^{2}+a_{22} h^{2}+a_{33} c^{2}+a_{44} I F R^{2}
\end{array}
$$

The values of the coefficients in the above equation depended on the significance of the first-order effect and the interactions. Just like other statistical tools, success of this DOE analysis depended on a careful and systematic data collection approach to avoid introduction of random and user errors. 
In order to quantify the error associated with measurement of the response variables, replicates were included in the test matrix. The center point of the matrix was replicated seven times, and six additional replicates were run throughout the design space.

Table 2. Central composite test matrix.

\begin{tabular}{|c|c|c|c|c|}
\hline Phase & $\begin{array}{c}\text { Spacing } \\
{[\mathrm{mm}]}\end{array}$ & $\begin{array}{c}\text { Height } \\
{[\mathrm{mm}]}\end{array}$ & $\begin{array}{c}\text { Chord } \\
{[\mathrm{mm}]}\end{array}$ & $\begin{array}{c}\text { Injection } \\
\text { Flow Ratio } \\
{[\%]}\end{array}$ \\
\hline Phase I & 25 & 3 & 12 & 0.0 \\
\hline Phase I & 25 & 3 & 12 & 1.0 \\
\hline Phase I & 25 & 3 & 24 & 0.0 \\
\hline Phase I & 25 & 3 & 24 & 1.0 \\
\hline Phase II & 25 & 4 & 18 & 0.5 \\
\hline Phase I & 25 & 5 & 12 & 0.0 \\
\hline Phase I & 25 & 5 & 12 & 1.0 \\
\hline Phase I & 25 & 5 & 24 & 0.0 \\
\hline Phase I & 25 & 5 & 24 & 1.0 \\
\hline Phase II & 30 & 3 & 18 & 0.5 \\
\hline Phase II & 30 & 4 & 12 & 0.5 \\
\hline Phase II & 30 & 4 & 18 & 0.0 \\
\hline Phase I & 30 & 4 & 18 & 0.5 \\
\hline Phase II & 30 & 4 & 18 & 1.0 \\
\hline Phase II & 30 & 4 & 24 & 0.5 \\
\hline Phase II & 30 & 5 & 18 & 0.5 \\
\hline Phase I & 35 & 3 & 12 & 0.0 \\
\hline Phase I & 35 & 3 & 12 & 1.0 \\
\hline Phase I & 35 & 3 & 24 & 0.0 \\
\hline Phase I & 35 & 3 & 24 & 1.0 \\
\hline Phase II & 35 & 4 & 18 & 0.5 \\
\hline Phase I & 35 & 5 & 12 & 0.0 \\
\hline Phase I & 35 & 5 & 12 & 1.0 \\
\hline Phase I & 35 & 5 & 24 & 0.0 \\
\hline Phase I & 35 & 5 & 24 & 1.0 \\
\hline
\end{tabular}

\section{Results}

\section{A. Baseline Case}

In order to assess the ability of the hybrid flow control to effect a reduction in flow separation, it was necessary to test the baseline uncontrolled case. Boundary-layer total pressure profiles were obtained using the translating pitot probe at various spanwise locations as indicated in Fig. 7. Immediately downstream of the normal shock impingement, the flow separated. This resulted in a layer of reversed flow. The pressure measurements made by the total pressure probe were lower than the static pressure measured at the wall surface. Although, the difference in the pressure was within the measurement uncertainty, the lower pressure measured by the probe was indicative of the large separation. The bump centerline static pressure in Fig. 8 shows the increase in static pressure across the normal shock. However, the static pressures were nearly constant from axial location of $\mathrm{x} \approx 27$ to $34 \mathrm{~cm}$, which shows a large separation exists at the aft end of the bump. 


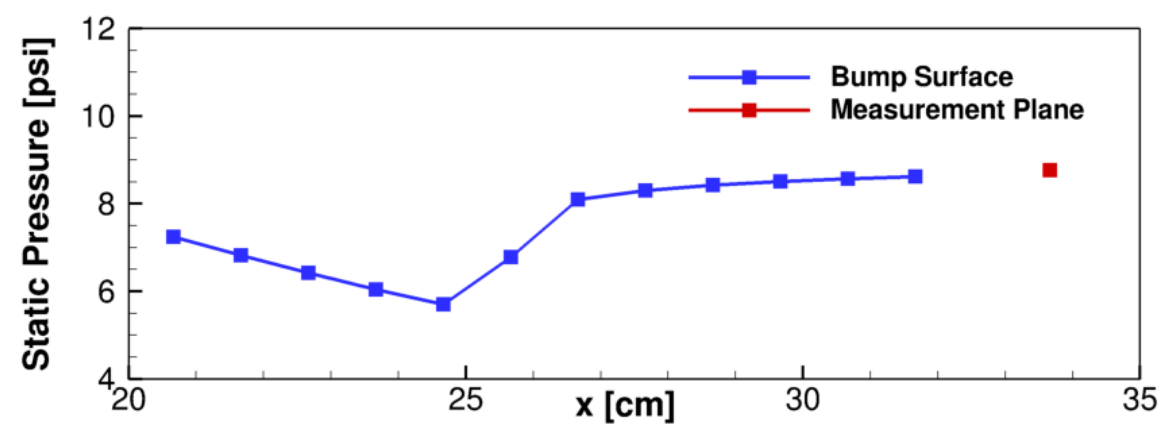

Figure 8. Centerline static pressure on the bump surface and at measurement plane.

To calculate the velocity within the reversed-flow region, the total pressure was assumed to be equal to that of the static pressure. The calculated velocities are shown in Fig. 10. From the contour plot of the normalized velocity magnitude shown in Fig. 9a we see that over the region from $\mathrm{z}=-1.5$ to $+1.5 \mathrm{~cm}$ the flow appears largely twodimensional. The line plot of the boundary layer velocity profiles in Fig. $9 \mathrm{~b}$ include the z-locations used to make the contour map as well as the profiles measured at $\mathrm{z}= \pm 3.5 \mathrm{~cm}$. The profiles at these outer measurement locations have smaller boundary-layer and reversed-flow thicknesses indicating 3-dimensionality of the flowfield even for the baseline configuration. The profiles at $\mathrm{z}= \pm 3.5 \mathrm{~cm}$ also show strong symmetry for the baseline flowfield. From these profiles, the boundary-layer thickness for the baseline configuration was found to be $2.89 \mathrm{~cm}$, and the reversed-flow thickness, $0.973 \mathrm{~cm}$.

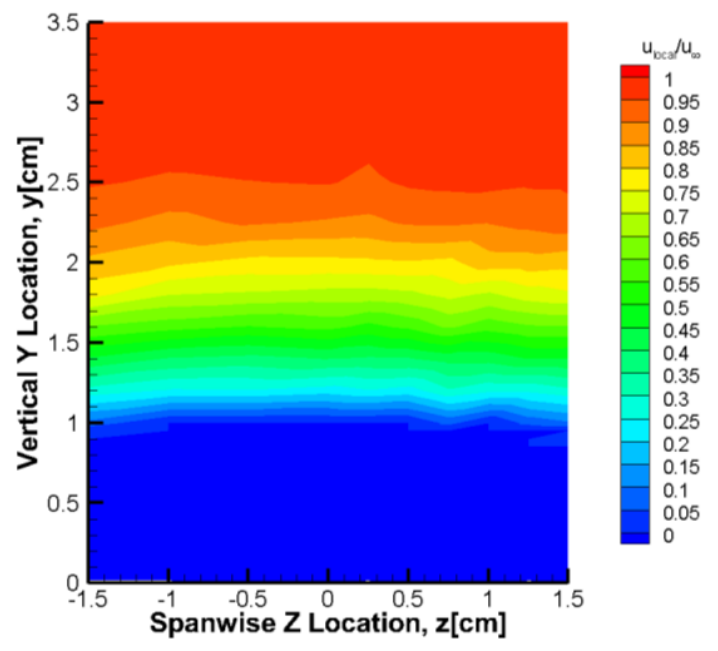

(a)

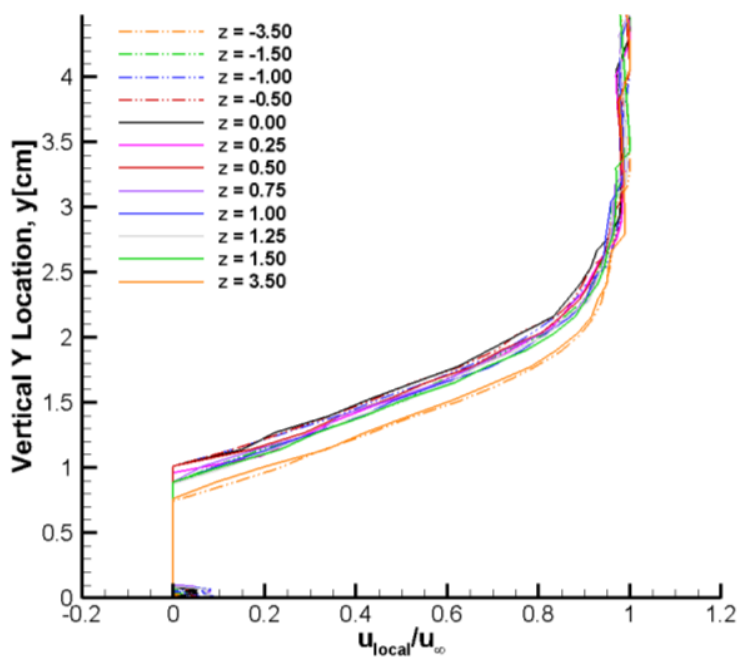

(b)

Figure 9. Velocity distribution for the baseline configuration. a) Normalized velocity contours. b) Boundary-layer normalized velocity profiles.

Profiles were also measured in the corners at a 45-degree angle. Because the outermost static taps were located at $\mathrm{z}= \pm 3.5 \mathrm{~cm}$, the measured profiles are shown in Fig. 10 as normalized total pressure. Agreement between the profiles for positive and negative $\mathrm{z}$ indicates a symmetric flowfield. 


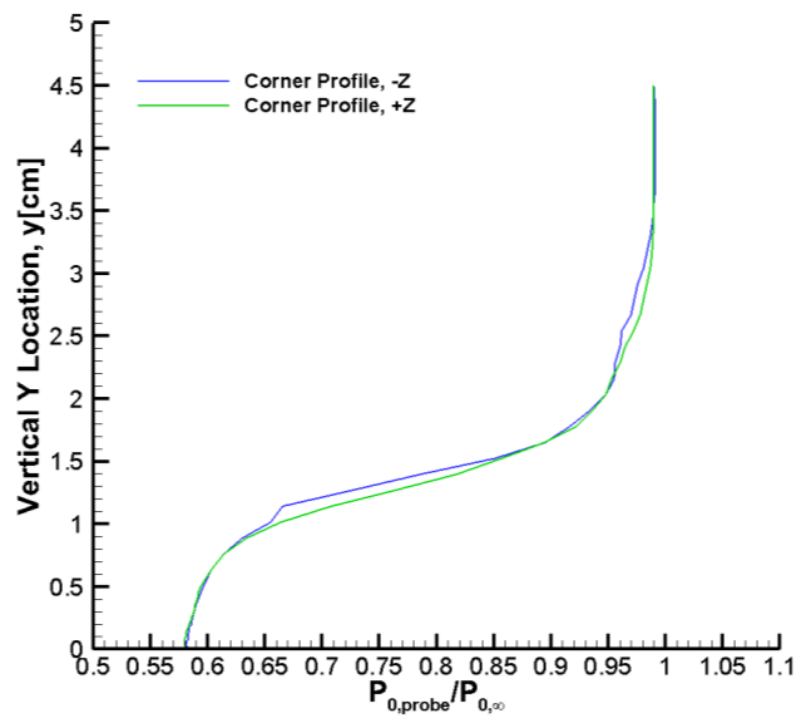

Figure 10. Normalized total pressure profiles in the corner, measured at a 45-degree angle.

\section{B. Injection Only}

During the analysis of the Phase I data, injection flow rate was seen to have a large influence on the effectiveness of the devices. Because of this, an additional configuration was tested in Phase II with steady microjet injection flow control and no micro-ramps. For this configuration, the micro-jets were spaced $30 \mathrm{~mm}$ apart and the total injection flow ratio for the four ports was $1.0 \%$ of the tunnel mass flow rate.

The calculated velocities are shown in Fig. 11. The profiles at $\mathrm{z}= \pm 1.5 \mathrm{~cm}$ were in line with the injection ports. The effect of the injection can be seen at those locations in the contour plot in Fig. 11a as a slight decrease in reversed-flow thickness (bowing down of the blue contour levels) and increase in the boundary-layer thickness (bowing up of the orange contour levels). As in the baseline case, the profiles at $\mathrm{z}= \pm 3.5 \mathrm{~cm}$ for the injection only case, seen in Fig. 11b, have smaller boundary-layer and reversed-flow thicknesses. From these profiles, the spanaveraged boundary-layer thickness for the injection only configuration was found to be $2.80 \mathrm{~cm}$, and the spanaveraged reversed-flow thickness, $0.903 \mathrm{~cm}$, indicating a slight improvement over the uncontrolled case.

The corner profile for the injection only case is compared to the baseline corner profile in Fig. 12. Because the profile is measured at an angle, part of the profile is influenced by the outermost micro-jet. The micro-jet is positioned at $\mathrm{z}=4.5 \mathrm{~cm}$ and is seen to energize the flow in the region directly downstream of injection, which corresponds to $y=3.0 \mathrm{~cm}$ in the profile shown. The pressurized air leads to local pressure ratios greater than 1.0. 




(a)

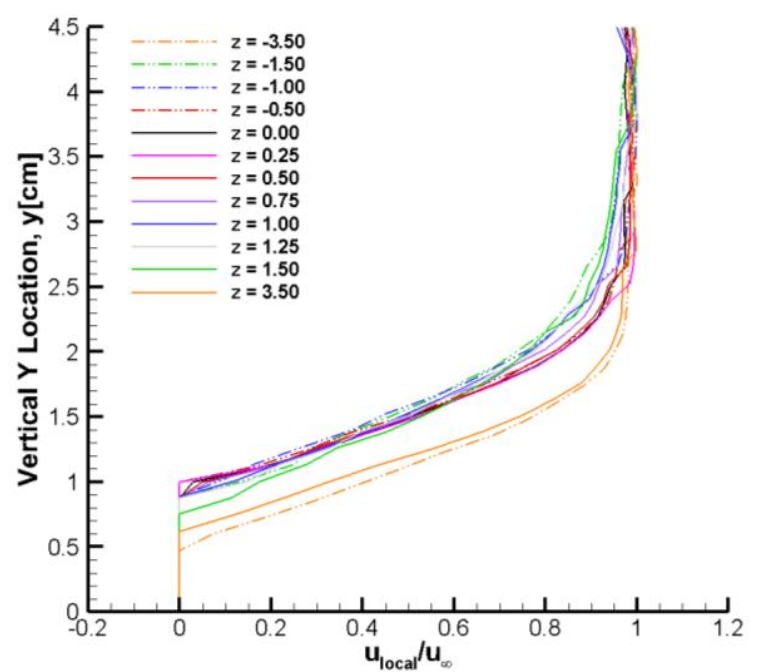

(b)

Figure 11. Velocity distribution for the injection only configuration. a) Normalized velocity contours. b) Boundary-layer normalized velocity profiles.

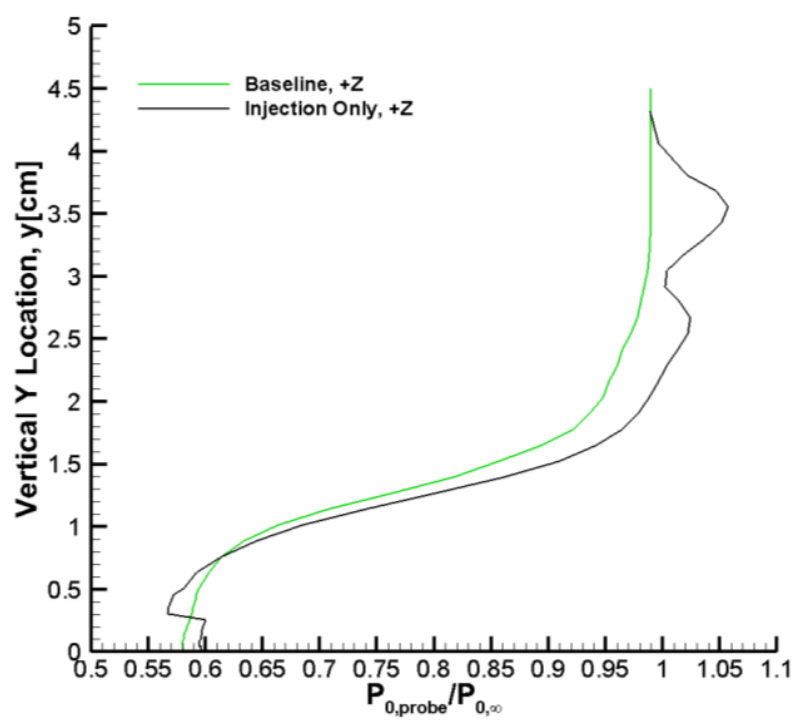

Figure 12. Normalized total pressure profiles in the corner, measured at a 45-degree angle. 


\section{DOE Analysis}

The measured boundary-layer thickness and reversed-flow thickness for each configuration are shown in Table 3. To determine the pure error of the measurements, points were replicated. All response values in the table are span averaged.

Table 3. Measured response variable values.

\begin{tabular}{|c|c|c|c|c|c|c|}
\hline Phase & $\begin{array}{c}\text { Spacing } \\
{[\mathrm{mm}]}\end{array}$ & $\begin{array}{l}\text { Height } \\
{[\mathrm{mm}]}\end{array}$ & $\begin{array}{l}\text { Chord } \\
{[\mathrm{mm}]}\end{array}$ & $\begin{array}{c}\text { Injection } \\
\text { Flow Ratio } \\
{[\%]}\end{array}$ & $\begin{array}{c}\text { Boundary-Layer } \\
\text { Thickness } \\
{[\mathrm{cm}]}\end{array}$ & $\begin{array}{c}\text { Reversed-flow } \\
\text { Thickness } \\
{[\mathrm{cm}]}\end{array}$ \\
\hline Phase I & \multicolumn{4}{|c|}{ Baseline } & 2.89 & 0.973 \\
\hline Phase II & \multicolumn{4}{|c|}{ Baseline with Injection } & 2.80 & 0.903 \\
\hline Phase I & 25 & 3 & 12 & 0.0 & 2.88 & 0.958 \\
\hline Phase I & 25 & 3 & 12 & 1.0 & 3.00 & 0.959 \\
\hline Phase I & 25 & 3 & 24 & 0.0 & 2.93 & 0.681 \\
\hline Phase I & 25 & 3 & 24 & 1.0 & 2.99 & 0.930 \\
\hline Phase II & 25 & 4 & 18 & 0.5 & 2.75 & 0.624 \\
\hline Phase I & 25 & 5 & 12 & 0.0 & 2.95 & 0.624 \\
\hline Phase I & 25 & 5 & 12 & 1.0 & 2.20 & 0.483 \\
\hline Phase I & 25 & 5 & 24 & 0.0 & 2.80 & 0.338 \\
\hline Phase I & 25 & 5 & 24 & 1.0 & 2.27 & 0.389 \\
\hline Phase II & 30 & 3 & 18 & 0.5 & 2.52 & 0.896 \\
\hline Phase II & 30 & 4 & 12 & 0.5 & 2.75 & 0.735 \\
\hline Phase II & 30 & 4 & 18 & 0.0 & 3.21 & 0.714 \\
\hline Phase I & 30 & 4 & 18 & 0.5 & 2.70 & 0.810 \\
\hline Phase II & 30 & 4 & 18 & 1.0 & 2.50 & 0.541 \\
\hline Phase II & 30 & 4 & 24 & 0.5 & 3.11 & 0.629 \\
\hline Phase I & 30 & 5 & 18 & 0.5 & 2.51 & 0.621 \\
\hline Phase I & 35 & 3 & 12 & 0.0 & 3.03 & 0.935 \\
\hline Phase I & 35 & 3 & 12 & 1.0 & 2.97 & 0.738 \\
\hline Phase I & 35 & 3 & 24 & 0.0 & 2.98 & 0.869 \\
\hline Phase I & 35 & 3 & 24 & 1.0 & 2.95 & 0.755 \\
\hline Phase II & 35 & 4 & 18 & 0.5 & 2.86 & 0.836 \\
\hline Phase I & 35 & 5 & 12 & 0.0 & 2.92 & 0.833 \\
\hline Phase I & 35 & 5 & 12 & 1.0 & 2.41 & 0.425 \\
\hline Phase I & 35 & 5 & 24 & 0.0 & 3.00 & 0.845 \\
\hline Phase I & 35 & 5 & 24 & 1.0 & 2.43 & 0.275 \\
\hline \multicolumn{7}{|c|}{ Replicates } \\
\hline Phase II & \multicolumn{4}{|c|}{ Baseline } & 3.40 & 0.962 \\
\hline Phase I & 25 & 3 & 12 & 0.0 & 2.89 & 0.954 \\
\hline Phase II & 25 & 5 & 24 & 0.0 & 2.98 & 0.322 \\
\hline Phase I & 25 & 5 & 24 & 1.0 & 2.15 & 0.444 \\
\hline Phase I & 30 & 4 & 18 & 0.5 & 2.47 & 0.766 \\
\hline Phase I & 30 & 4 & 18 & 0.5 & 2.87 & 0.786 \\
\hline Phase I & 30 & 4 & 18 & 0.5 & 2.77 & 0.796 \\
\hline Phase I & 30 & 4 & 18 & 0.5 & 2.42 & 0.785 \\
\hline Phase II & 30 & 4 & 18 & 0.5 & 2.85 & 0.725 \\
\hline Phase II & 30 & 4 & 18 & 0.5 & 2.73 & 0.809 \\
\hline Phase II & 30 & 5 & 18 & 0.5 & 2.71 & 0.628 \\
\hline Phase I & 35 & 5 & 24 & 1.0 & 2.34 & 0.327 \\
\hline Phase II & 35 & 5 & 24 & 1.0 & 2.29 & 0.207 \\
\hline
\end{tabular}




\section{Boundary-layer Thickness $(\delta)$}

A response surface was created to model the variation of the boundary-layer thickness within the design space. The response surface equation for boundary-layer thickness in terms of the factor variables is:

$$
\begin{array}{r}
\delta=a_{o}+a_{1} s+a_{2} h+a_{3} c+a_{4} I F R+a_{12} s h+a_{13} s c+a_{14} s I F R+a_{23} h c+a_{24} h I F R+a_{34} c I F R+ \\
a_{11} s^{2}+a_{22} h^{2}+a_{33} c^{2}+a_{44} I F R^{2}
\end{array}
$$

Coefficients were calculated based on the measured span-averaged responses shown in Table 3. The resulting coefficients and p-value for each factor, along with the model and lack of fit (LOF) p-values are shown in Table 4. Three different models are presented (factorial, quadratic 1, and quadratic 2). Blank cells in the table indicate that the factors corresponding to those coefficients were not considered for that model. Cells with dashes indicate that the factor was considered, but was dropped from the model.

The coefficients are shown in terms of both actual and coded factors. The actual coefficients can be used in Eq. 1 with the values of the factor variables in engineering units (in this case, $s, h$, and $c$ in $\mathrm{mm}$, and IFR as percent of total tunnel flow rate). The coded coefficients are used in the equation with the factor variables transformed into coded units where the range of the variable is mapped to a range from -1 to +1 . For example, $s=25 \mathrm{~mm}$ is $s=-1$ in coded units, $s=30 \mathrm{~mm}$ is $s=0$, and $s=35 \mathrm{~mm}$ is $s=+1$. Coded coefficients are useful because they are proportional to the magnitude of the observed effects. That is, if a coded coefficient is large, that factor or interaction had a large influence on the measured response.

To determine which factors to retain in the model, for each factor we use the null hypothesis that there is no effect from that factor. The probability of observing the measured values of the response variable is calculated assuming the null hypothesis is true. If the probability is small, then it is concluded that the factor influences the response. The cutoff value used for this analysis was $p=0.05$.

It may be unintuitive then that some coefficients shown in Table 5 have a large p-value, yet were retained. These factors were included to preserve model hierarchy, meaning that the lower order (parent) terms have been included in the model for all higher order terms. For instance the $a_{3}$ coefficient in the quadratic 2 model is retained because it is the parent term for the $\mathrm{a}_{33}$ coefficient. If hierarchy is not preserved then predictions based on the actual and coded equations will not match.

A p-value and lack of fit are also shown for each model. If the model p-value is small, then the terms in the model have a significant effect on the response. If lack of fit is small, the residuals for the model are large compared to the pure error, and we conclude that the model does not fit the data well. The cutoff for lack of fit was 0.1.

\begin{tabular}{|c|c|c|c|c|c|c|c|c|c|}
\hline & \multicolumn{3}{|c|}{ Factorial } & \multicolumn{3}{|c|}{ Quadratic 1 } & \multicolumn{3}{|c|}{ Quadratic 2} \\
\hline & $\mathrm{p}=<0.0001$ & \multicolumn{2}{|c|}{$\mathrm{LOF}=0.5468$} & \multicolumn{2}{|c|}{$\mathrm{p}=<0.0001$} & $\mathrm{LOF}=0.6606$ & \multicolumn{2}{|c|}{$\mathrm{p}=<0.0001$} & $\mathrm{LOF}=0.6820$ \\
\hline & coef $_{\text {actual }}$ & coef $_{\text {coded }}$ & $\mathrm{p}$-value & coef $_{\text {actual }}$ & coef $_{\text {coded }}$ & $\mathrm{p}$-value & coef $_{\text {actual }}$ & coef $_{\text {coded }}$ & $\mathrm{p}$-value \\
\hline $\mathrm{a}_{0}$ & 2.954 & 2.79 & -- & 6.316 & 2.73 & -- & 4.056 & 2.72 & -- \\
\hline $\mathrm{a}_{1}$ & -- & -- & -- & -0.236 & 0.043 & 0.1421 & -- & -- & -- \\
\hline $\mathrm{a}_{2}$ & $-1.761 \mathrm{E}-04$ & -0.16 & $<0.0001$ & $-1.110 \mathrm{E}-03$ & -0.16 & $<0.0001$ & $-2.482 \mathrm{E}-03$ & -0.16 & $<0.0001$ \\
\hline $\mathrm{a}_{3}$ & -- & -- & -- & -- & -- & -- & -0.130 & 4.298E-03 & 0.8844 \\
\hline $\mathrm{a}_{4}$ & 0.920 & -0.17 & $<0.0001$ & 0.952 & -0.17 & $<0.0001$ & 0.959 & -0.17 & $<0.0001$ \\
\hline$a_{12}$ & -- & -- & -- & -- & -- & -- & -- & -- & -- \\
\hline$a_{13}$ & -- & -- & -- & -- & -- & -- & -- & -- & -- \\
\hline$a_{14}$ & -- & -- & -- & -- & -- & -- & -- & -- & -- \\
\hline$a_{23}$ & -- & -- & -- & -- & -- & -- & -- & -- & -- \\
\hline $\mathrm{a}_{24}$ & -0.314 & -0.16 & $<0.0001$ & -0.325 & -0.16 & $<0.0001$ & -0.324 & -0.16 & $<0.0001$ \\
\hline$a_{34}$ & -- & -- & -- & -- & -- & -- & -- & -- & -- \\
\hline$a_{11}$ & & & & $4.08 \mathrm{E}-03$ & 0.10 & 0.0494 & -- & -- & -- \\
\hline $\mathrm{a}_{22}$ & & & & -- & -- & -- & -- & -- & -- \\
\hline$a_{33}$ & & & & -- & -- & -- & $3.628 \mathrm{E}-03$ & 0.13 & 0.0129 \\
\hline$a_{44}$ & & & & -- & -- & -- & -- & -- & -- \\
\hline
\end{tabular}

Table 4. Coefficients for three boundary-layer thickness models

12

American Institute of Aeronautics and Astronautics 
Comparing the models presented in Table 4, each of them can be said statistically to adequately explain the variations in boundary-layer thickness due to the factor variables. Interestingly, the factorial model only depends upon the micro-ramp height, the injection flow ratio, and the interaction between these two variables.

Slightly better fit is obtained if we expand to a quadratic model. The model with the highest possible value for lack of fit, quadratic 2, contains each of the terms from the factorial model plus the effects of chord length and its squared term. The quadratic 1 model has a lack of fit value nearly as large, but includes inter-ramp spacing and its squared term while neglecting the chord length. In both cases, the coded coefficients show a tendency for the factor variable to increase the boundary-layer thickness. Even though the lack of fit is slightly better for quadratic 2, considering the system physically the model in terms of the spacing can be explained as follows: when the spacing increases, the vortices are unable to influence the entire span, and therefore the span-averaged boundary-layer thickness increases. For this reason, the rest of the analysis in this section will be based on the quadratic 1 model.

Figure 13 shows how the predicted values of the span-averaged boundary-layer thickness compare to the measured values for every configuration using the quadratic 1 model. The R-squared value for the model is 0.797 . Because the testing was done in two phases, a blocking term was introduced. Blocking is the process of grouping configurations that are similar to one another (in this case by Phase I and II) so that any unknown effects due to the known change are accounted for in the blocking variable. Block effects are considered large when the mean square (an estimate of the variance) for the block term is three to four times larger than the mean square of the model residuals. In this case, the block mean square value was 0.061 , and the model residual mean square was 0.019 , indicating the block effect for boundary layer thickness was large. The model residual consists of the model lack of fit and the pure error. The model lack of fit is shown in Table 5. Pure error is a measurement of the precision of the response values for the replicates. The mean square of the pure error for boundary-layer thickness was 0.021 . Since the mean square is an estimate of the variance, the standard deviation of the replicated points was approximately 0.145 .

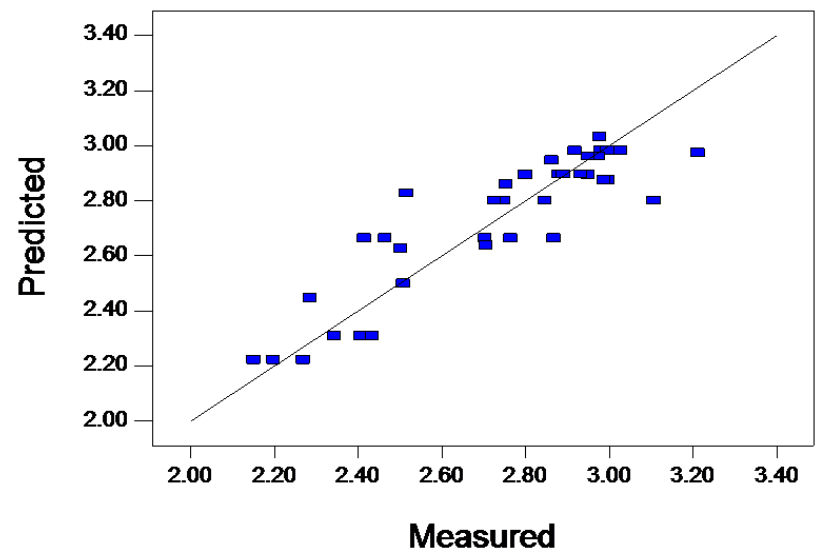

Figure 13. Predicted values of span-averaged boundary-layer thickness versus measured values.

Figure 14 shows the variation in boundary-layer thickness one factor at a time with the other three factors held at their central values (coded value $=0$ ). The red points in the figure represent the measured values, and the dashed lines on either side of the response curve indicate the confidence interval of the model. The effect of spacing can be seen in Fig. 14a. Due to the quadratic term in the model, the response surface is a minimum at a spacing in the middle of the design range. The seven points seen at $s=30 \mathrm{~mm}$ are the seven replicates of the center point of the design space. Factors $h$ and IFR (seen in Figs. 14b and 14c respectively) are linear, and the response surface is at a minimum for $h=5 \mathrm{~mm}$ and $I F R=1.0 \%$. The $h$-IFR interaction term in the model indicates that the slope of these curves changes through the design space. 




(a)

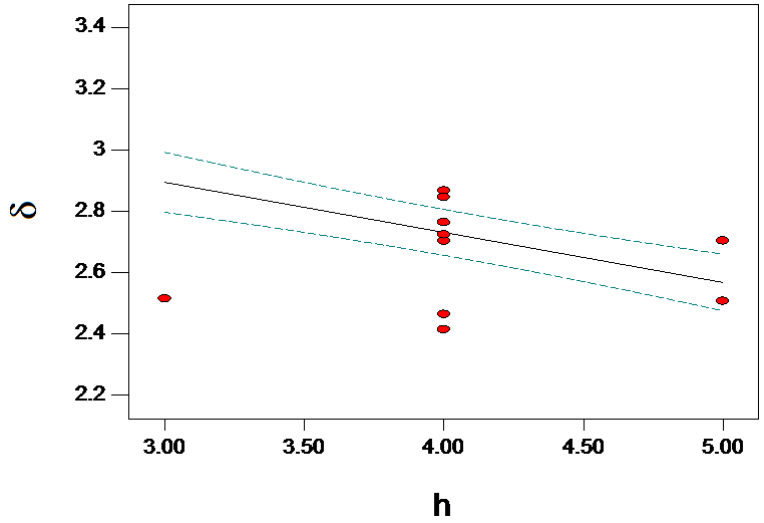

(b)

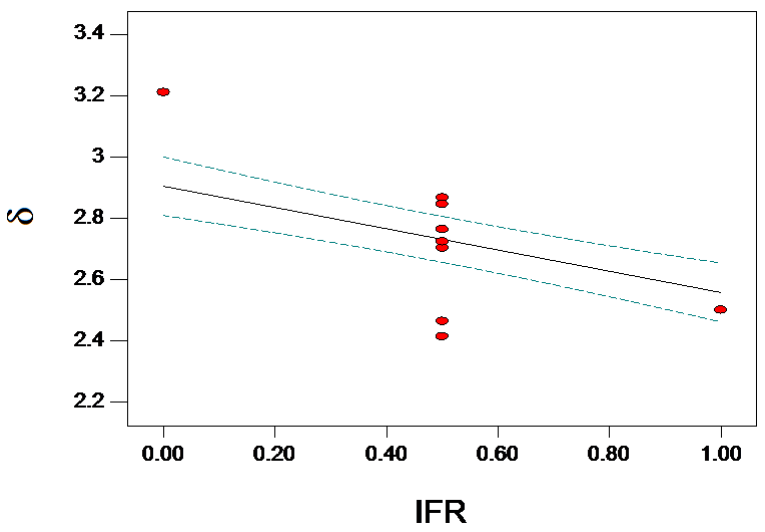

(c)

Figure 14. One factor at a time plots of boundary-layer thickness. All other factors held at their central level. a) Effect of inter-ramp spacing. b) Effect of micro-ramp height. c) Effect of injection flow ratio.

The $h$-IFR interaction can be seen in the contour plot of boundary layer thickness shown in Fig. 15. These contour are at the plane $s=30 \mathrm{~mm}$. In the figure, the red dots indicate locations in the design space where data exists. The value of the chord length is unspecified because it does not appear in the response surface equation (quadratic 1). The contour plot indicates that having either large micro-ramp height or large injection flow ratio is insufficient to substantially decrease the boundary-layer thickness. Only when both are increased in tandem does the boundary-layer thickness improve.

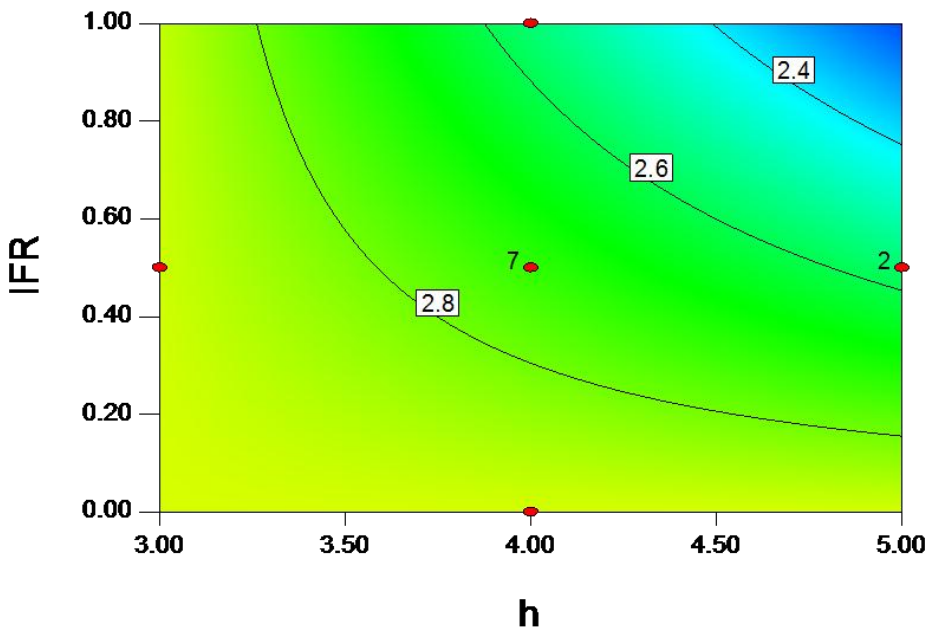

Figure 15. Contour of boundary-layer thickness for the $h-I F R$ interaction. Shown for $s=30 \mathrm{~mm}$.

14

American Institute of Aeronautics and Astronautics 


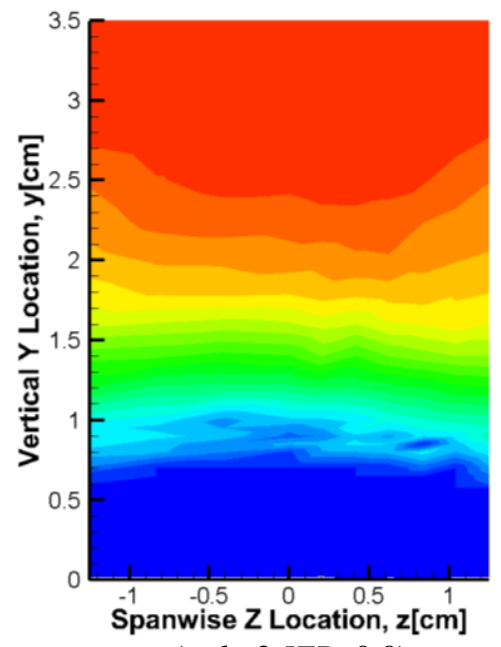

(a: $h=3, I F R=0.0)$



(c: $h=5, I F R=0.0)$

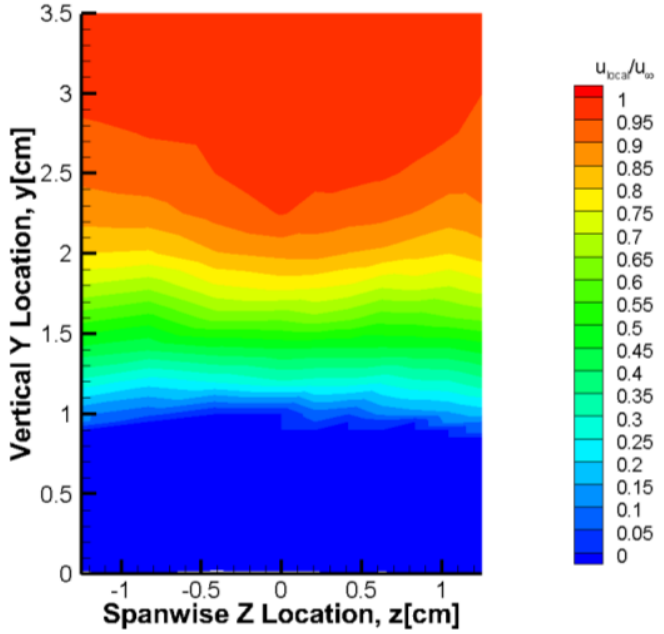

(b: $h=3, I F R=1.0$ )

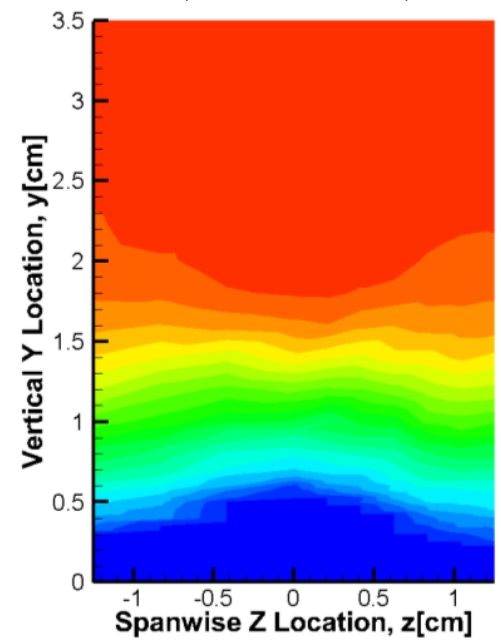

$(\mathrm{d}: h=5, I F R=1.0)$

Figure 16. Normalized velocity contour profiles for the $h-I F R$ interaction with $s=25 \mathrm{~mm}$ and $c=24 \mathbf{m m}$.

To understand how the micro-ramp height and injection flow ratio interacted to affect the boundary-layer thickness, contour plots of normalized velocity for four configurations are shown in Fig. 16. Each of the configurations had $s=25 \mathrm{~mm}$ and $c=24 \mathrm{~mm}$. In Fig. 16a contours are shown for the $h=3 \mathrm{~mm}, I F R=0.0 \%$ configuration. The effects generally appear similar to what was seen earlier for the injection only configuration: a slight thickening of the boundary layer in line with the devices, which are located at $\mathrm{z}= \pm 1.25 \mathrm{~cm}$, and thinning between the devices. Adding injection $(I F R=1.0 \%)$ to the $h=3 \mathrm{~mm}$ configuration increased the boundary-layer thickness slightly, as seen in Fig. 16b. For the $h=5 \mathrm{~mm}, I F R=0.0 \%$ configuration the location of the micro-ramp vortex is clearly visible in the contours seen in Fig. 16c. On a span-averaged basis, the boundary layer was slightly thinner than the baseline case because of the thinning between the devices, but the boundary layer thickness was highly non-uniform across the span. In Fig. 16d, the effect of adding 1.0\% flow injection ratio to the large ramps is seen as an increase in the uniformity across the span. The high momentum flow in the micro-jet reenergized the flow in the velocity deficit region created by the vortex, and the boundary layer was thinned in all measured $\mathrm{z}$ locations from -1.25 to $+1.25 \mathrm{~cm}$. 


\section{Reversed-flow Thickness (RFT)}

A response surface was created to model the variation of the reversed-flow thickness within the design space. As will be shown, a quadratic model was insufficient to fit the data for span-averaged reversed-flow thickness. The central composite design contains enough data to include up to 8 second order interactions. Therefore, the response surface equation for reversed-flow thickness in terms of the factor variables is:

$$
\begin{array}{r}
R F T=a_{o}+a_{1} s+a_{2} h+a_{3} c+a_{4} I F R+a_{12} s h+a_{13} s c+a_{14} s I F R+a_{23} h c+a_{24} h I F R+ \\
a_{34} C I F R+a_{11} s^{2}+a_{22} h^{2}+a_{33} c^{2}+a_{44} I F R^{2}+a_{123} s h c+a_{124} s h I F R+a_{134} S C I F R+a_{234} h c I F R+ \\
a_{112} s^{2} h+a_{113} s^{2} c+a_{114} s^{2} I F R+a_{122} h^{2}
\end{array}
$$

Coefficients were calculated based on the measured span-averaged responses shown in Table 3. The resulting coefficients and p-value for each factor, along with the model and lack of fit (LOF) p-values are shown in Table 5. Three different models are presented (factorial, quadratic, and higher order interactions). As before, blank cells in the table indicate that the factors corresponding to those coefficients were not considered for that model. Cells with

\begin{tabular}{|c|c|c|c|c|c|c|c|c|c|}
\hline & \multicolumn{3}{|c|}{ Factorial } & \multicolumn{3}{|c|}{ Quadratic } & \multicolumn{3}{|c|}{ Higher Order Interactions } \\
\hline & \multicolumn{2}{|c|}{$\mathrm{p}=<0.0001$} & $\mathrm{LOF}=0.0016$ & \multicolumn{2}{|c|}{$\mathrm{p}=<0.0001$} & $\mathrm{LOF}=0.0079$ & \multicolumn{2}{|c|}{$\mathrm{p}=<0.0001$} & $\mathrm{LOF}=0.1672$ \\
\hline & coef $_{\text {actual }}$ & coef $_{\text {coded }}$ & $\mathrm{p}$-value & coef $_{\text {actual }}$ & coef $_{\text {coded }}$ & $\mathrm{p}$-value & coef $_{\text {actual }}$ & coef $f_{\text {coded }}$ & $\mathrm{p}$-value \\
\hline $\mathrm{a}_{0}$ & 1.836 & 0.710 & -- & 1.84 & 0.760 & -- & 4.056 & 0.76 & -- \\
\hline $\mathrm{a}_{1}$ & -0.014 & 0.023 & 0.1931 & -0.016 & 0.021 & 0.1411 & -- & 0.11 & 0.0009 \\
\hline $\mathrm{a}_{2}$ & -0.403 & -0.170 & $<0.0001$ & -0.406 & -0.160 & $<0.0001$ & $-2.482 \mathrm{E}-03$ & -0.16 & $<0.0001$ \\
\hline $\mathrm{a}_{3}$ & -0.011 & -0.065 & 0.0008 & $-9.95 \mathrm{E}-03$ & -0.060 & 0.0002 & -0.130 & -0.054 & $<0.0001$ \\
\hline $\mathrm{a}_{4}$ & 1.528 & -0.073 & 0.0002 & 1.81 & -0.073 & $<0.0001$ & 0.959 & -0.073 & $<0.0001$ \\
\hline$a_{12}$ & $9.76 \mathrm{E}-03$ & 0.049 & 0.0117 & $9.88 \mathrm{E}-03$ & 0.049 & 0.0020 & -- & 0.045 & $<0.0001$ \\
\hline$a_{13}$ & -- & -- & -- & -- & -- & -- & -- & 0.028 & 0.0055 \\
\hline $\mathrm{a}_{14}$ & -0.041 & -0.100 & $<0.0001$ & -0.038 & -0.095 & $<0.0001$ & -- & -0.092 & $<0.0001$ \\
\hline$a_{23}$ & -- & -- & -- & -- & -- & -- & -- & -- & -- \\
\hline $\mathrm{a}_{24}$ & -0.112 & -0.056 & $<0.0001$ & -0.106 & -0.053 & 0.0010 & -0.324 & -0.064 & $<0.0001$ \\
\hline$a_{34}$ & -- & -- & -- & -- & -- & -- & -- & 0.022 & 0.0255 \\
\hline$a_{11}$ & & & & -- & -- & -- & -- & -- & -- \\
\hline$a_{22}$ & & & & -- & -- & -- & -- & $4.18 \mathrm{E}-03$ & 0.8463 \\
\hline$a_{33}$ & & & & -- & -- & -- & -- & -- & -- \\
\hline $\mathrm{a}_{44}$ & & & & -0.390 & -0.097 & 0.0003 & -- & -0.097 & $<0.0001$ \\
\hline$a_{123}$ & & & & & & & -- & -- & -- \\
\hline$a_{124}$ & & & & & & & -- & -0.022 & 0.0238 \\
\hline$a_{134}$ & & & & & & & -- & -0.034 & 0.0011 \\
\hline $\mathrm{a}_{234}$ & & & & & & & -- & -- & -- \\
\hline$a_{112}$ & & & & & & & -- & -- & -- \\
\hline $\mathrm{a}_{113}$ & & & & & & & -- & -- & -- \\
\hline$a_{114}$ & & & & & & & -- & -- & -- \\
\hline$a_{122}$ & & & & & & & -- & -0.090 & 0.0052 \\
\hline
\end{tabular}
dashes indicate that the factor was considered, but was dropped from the model.

Table 5. Coefficients for three reversed-flow thickness models 
Comparing the models presented in Table 5, only the model which includes higher order interactions can be said statistically to adequately explain the variations in reversed-flow thickness due to the factor variables. This illustrates why the full factorial test matrix tested in Phase I was inadequate to generate a predictive model. The quadratic model also fails to pass the test for lack of fit which requires a value greater than 0.1 .

Figure 17 shows how the predicted values of the span-averaged reversed-flow thickness compare to the measured values for every configuration. The R-squared value for the model is 0.98 . As for the boundary-layer thickness analysis, a blocking term was introduced. In this case, the block mean square value was 0.056 , and the model residual mean square was 0.0015 , so our block effect for reversed-flow thickness was very large. The mean square of the pure error for reversed-flow thickness was $9.38 \mathrm{E}-4$. Since the mean square is an estimate of the variance, the standard deviation of the replicated points was approximately 0.031 .

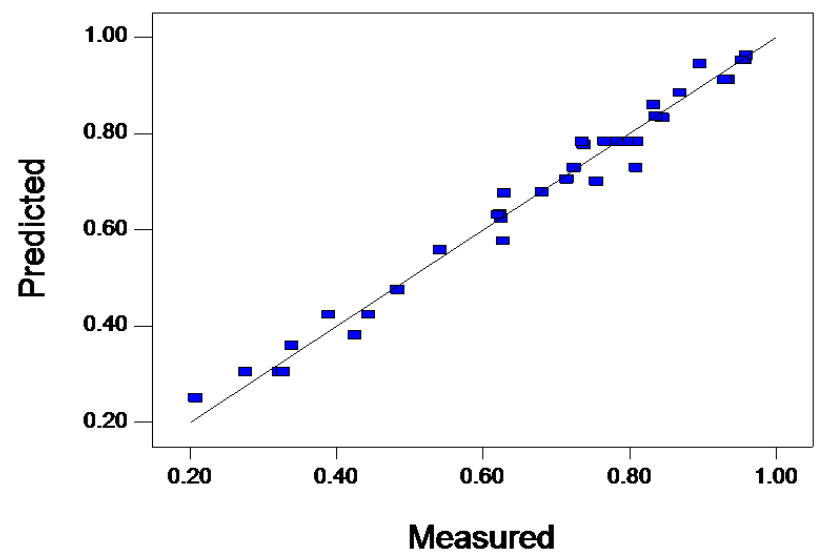

Figure 17. Predicted values of span-averaged reversed-flow thickness versus measured values.

Figure 18 shows the variation in reversed-flow thickness one factor at a time with the other factors held at their central values (coded value $=0$ ). The red points in the figure represent the measured values, and the dashed lines on either side of the response curve indicate the confidence interval of the model. The effect of spacing can be seen in Fig. 18a. When all of the other factors were at their central levels, reversed-flow thickness was a minimum for $s=$ $25 \mathrm{~mm}$. However, due to the complex nature of the response surface equation, this is not true throughout the entire design space. Again, seven points are seen at $s=30 \mathrm{~mm}$ which are the seven replicates of the center point of the design space. It is interesting to note the small range of reversed-flow thickness for these seven replicates, which corresponds to the small value for pure error reported above. Additionally, the confidence intervals are much closer to the prediction line for reversed-flow thickness than they were for boundary-layer thickness due to the small pure error. In terms of factors $h$ and $c$ (seen in Figs. 18b and 18c respectively) reversed-flow thickness was at a minimum at $h=5 \mathrm{~mm}$ and $c=24 \mathrm{~mm}$. This generally holds true through the design space as these large micro-ramps produce strong vortices that decrease the magnitude of the reversed-flow region. In the plot for injection flow ratio, Fig. 18d, the curvature is such that $I F R=0.0 \%$ and $I F R=1.0 \%$ result in smaller reversed-flow thicknesses than the intermediate value, $I F R=0.5 \%$, when all of the other factors are at their central levels. 




(a)

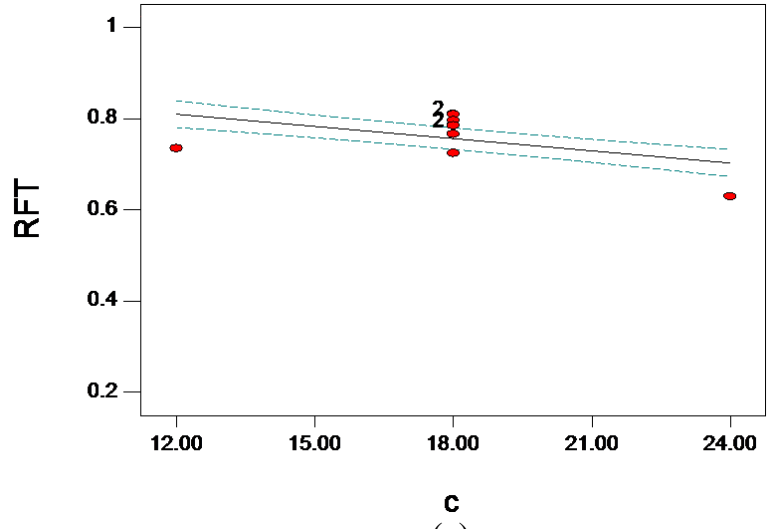

(c)

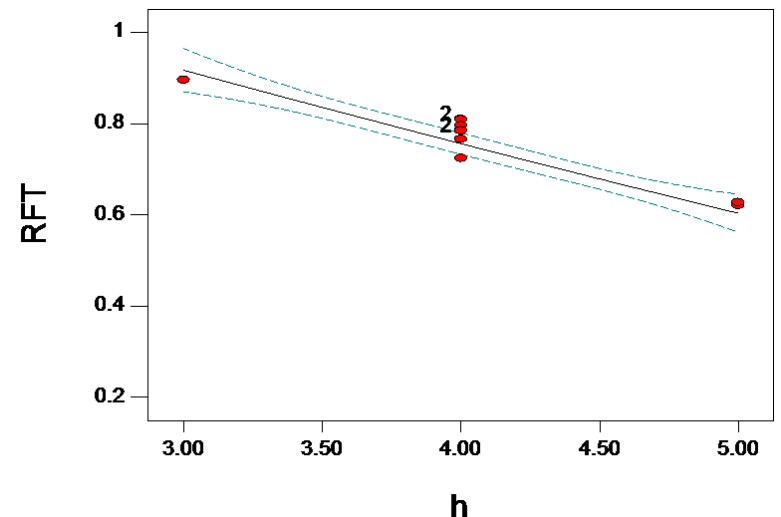

(b)

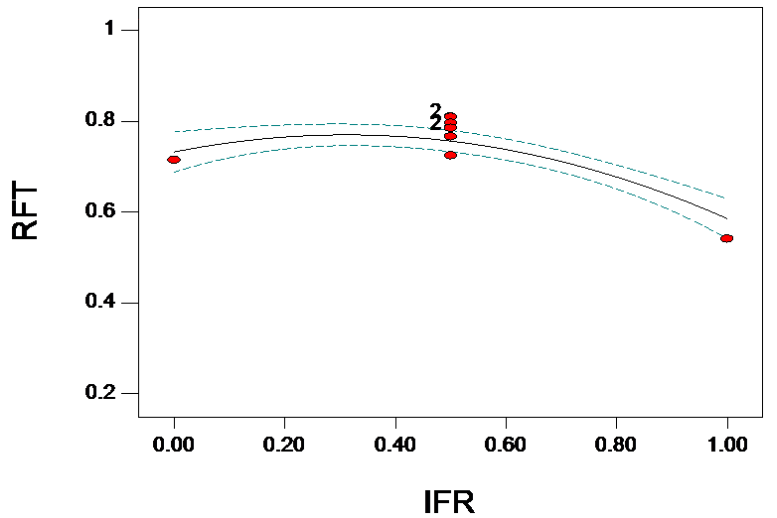

(d)

Figure 18. One factor at a time plots of reversed-flow thickness. All other factors held at their central level. a) Effect of inter-ramp spacing. b) Effect of micro-ramp height. c) Effect of micro-ramp chord length. d) Effect of injection flow ratio.

For the reversed-flow thickness response surface equation with many interaction terms, examining contour plots of the reversed-flow thickness can help visualize the response surface. Contours are shown in Fig. 19 of the predicted magnitude of the span-averaged reversed-flow thickness on the $s$ - $h$ plane. In the figure, the dots indicate locations in the design space where data exists. The contour values are labeled on the lines. The plots do not show the influence of the chord length, but it was the most direct: increased chord length had a small positive effect on the reversed-flow thickness. Each of the contour plots in Fig. 19 is displayed for $c=24 \mathrm{~mm}$.

In Fig. 19a the variations in reversed-flow thickness are seen for $I F R=0.0 \%$. The contours show that microramps without injection spaced $35 \mathrm{~mm}$ apart had very little impact on the reversed-flow thickness, regardless of height, compared to the baseline where $R F T=0.973 \mathrm{~cm}$. Reductions in reversed-flow thickness were larger at a smaller spacing, especially for larger $(h=5 \mathrm{~mm})$ micro-ramps. Previous work ${ }^{9}$ showed that closely spaced ramps had reduced vortex liftoff, allowing more mixing to occur, and this may explain some of the effect of spacing for micro-ramps without injection in the current study. Additionally, the region that the ramps influenced did not reach all the way across the span for ramps with the larger spacing.

For the case with $I F R=0.5 \%$, shown in Fig. 19b, the height of the ramps became more influential than the spacing. That is, large reversed-flow thickness was measured for $h=3 \mathrm{~mm}$ micro-ramps for the entire range of spacing. However, for $h=5 \mathrm{~mm}$, the lowest predicted reversed-flow thickness was still for $s=25 \mathrm{~mm}$.

For $I F R=1.0 \%$, shown in Fig. $19 \mathrm{c}$, it is similar to the $I F R=0.5 \%$ case in that there was more variation in reversed-flow thickness with height than spacing, and increasing height decreased reversed-flow thickness. Unlike the previous two cases, the minimum reversed-flow thickness occurred for $s=35 \mathrm{~mm}$.

To summarize, with only micro-ramps it was important to have the devices closely spaced so that the vortices could influence the entire span and follow a trajectory closer to the wall to allow for more mixing. In general larger height produced a greater reduction in reversed-flow thickness; however, at $I F R=0.0 \%$ it was better to have smaller 
height micro-ramps spaced closely together than micro-ramps with a large height spaced far apart. Once injection was included, height became key, and whether closely or widely spaced devices were better depended on the amount of flow injected. The interaction between spacing and injection flow rate was noted in the Phase I report ${ }^{1}$.

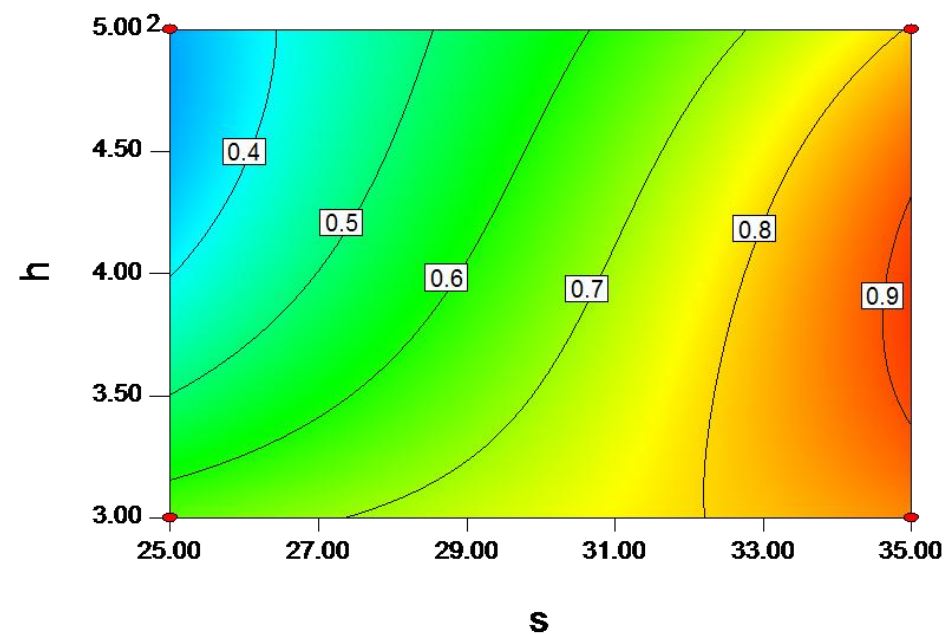

(a)

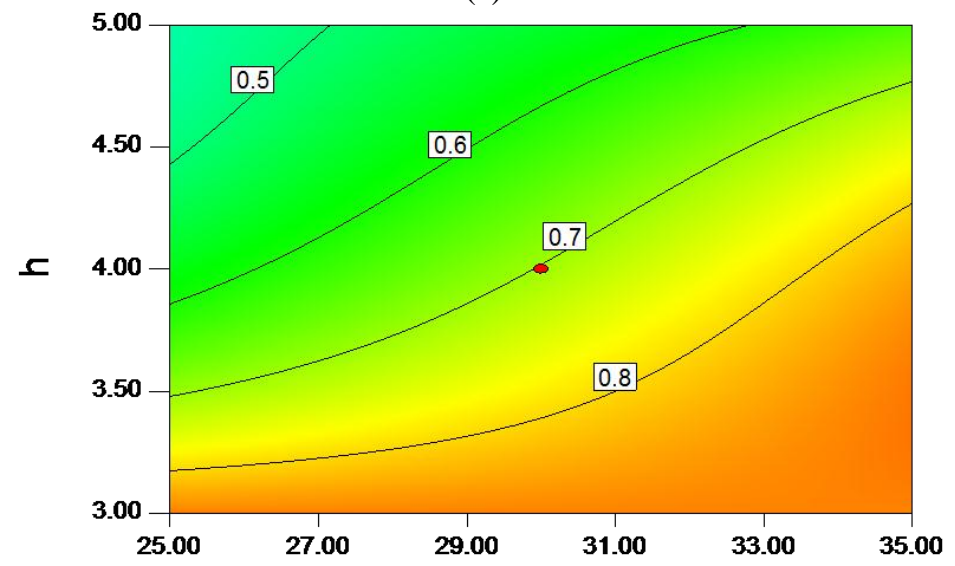

(b)

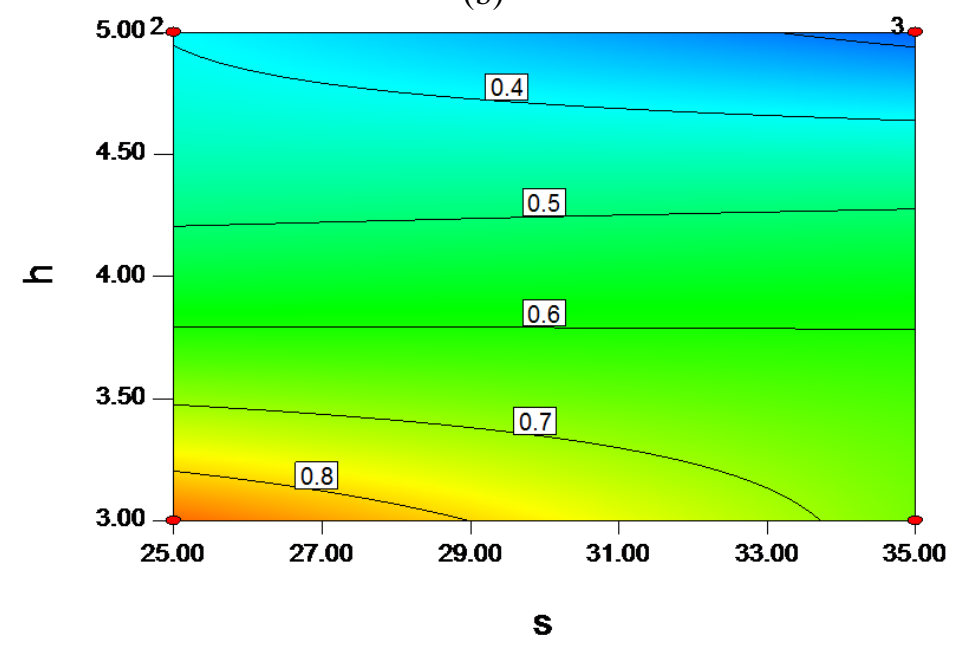

(c)

Figure 19. Contour of reversed-flow thickness for the $h$-s interaction. Shown for $c=24 \mathrm{~mm}$. a) $I F R=0.0 \%$. b) $I F R=$ $0.5 \%$. c) $I F R=1.0 \%$.

19

American Institute of Aeronautics and Astronautics 


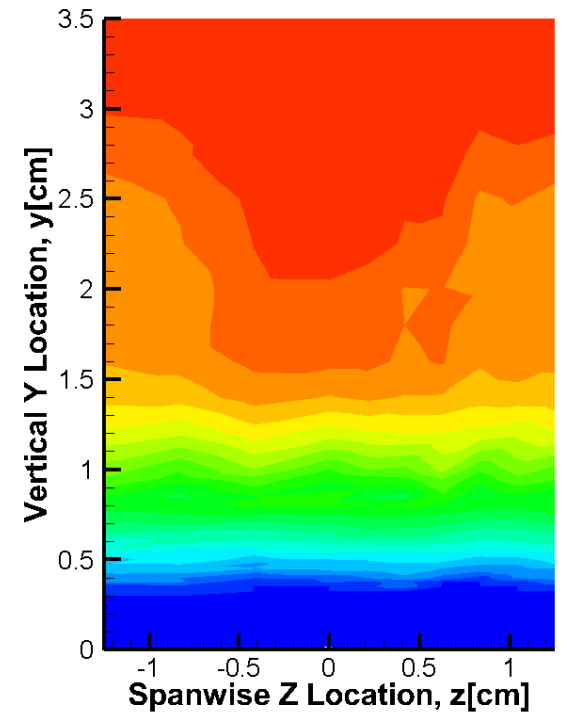

(a: $s=25, I F R=0.0)$

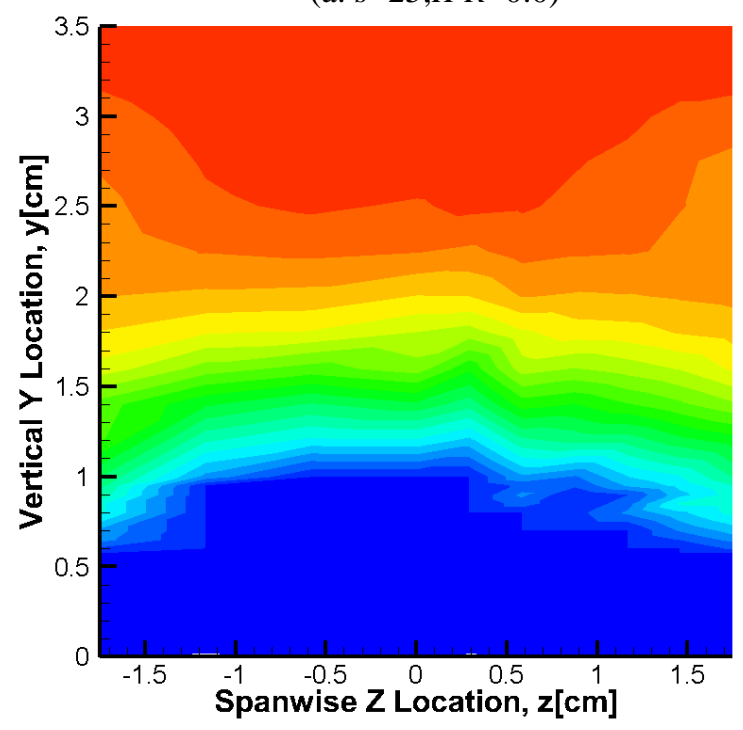

(c: $s=35, I F R=0.0$ )

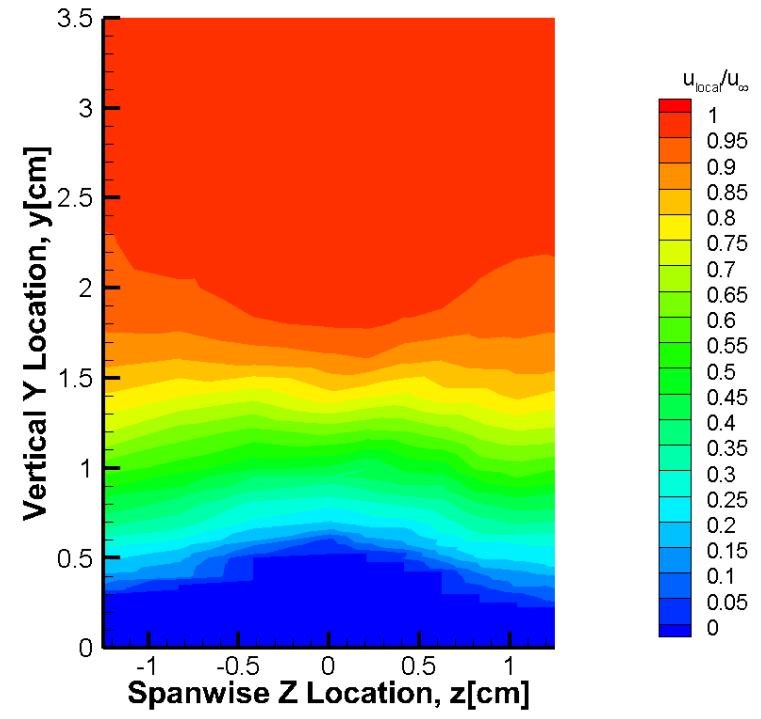

(b: $s=25, I F R=1.0$ )

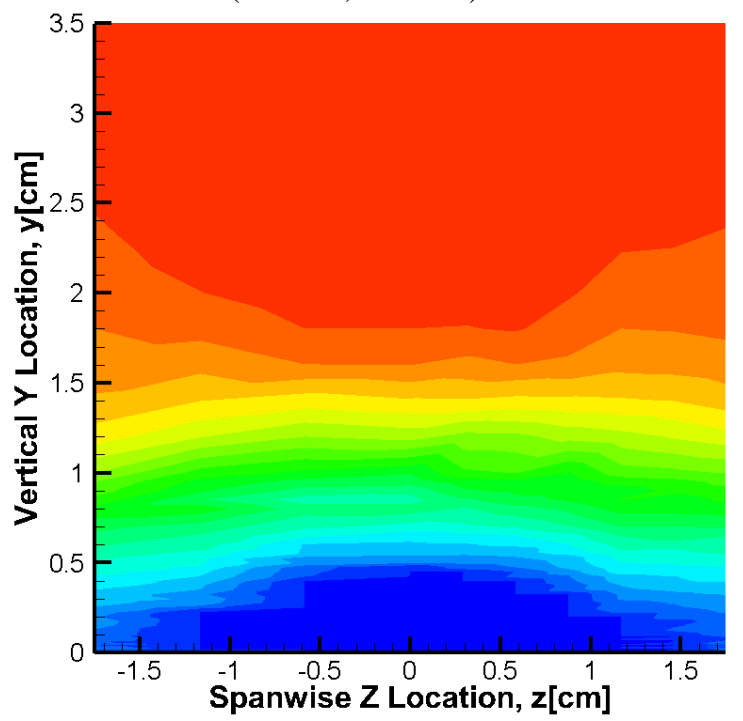

(d: $s=35, I F R=1.0)$

Figure 20. Normalized velocity contour profiles for the $s-I F R$ interaction with $h=5 \mathbf{m m}$ and $c=24 \mathbf{m m}$.

Examining the normalized velocity profiles at $h=5 \mathrm{~mm}$ and $c=24 \mathrm{~mm}$ for the four combinations of minimum and maximum spacing and injection flow ratio can help clarify the $s$-IFR interaction, and the velocity contours are shown in Fig. 20. Figures 20a and 20d represented the best of the measured hybrid flow control configurations (though as will be discussed in the next section, perhaps not the best possible configurations). The contours for $s=$ $25 \mathrm{~mm}$ and $I F R=0.0 \%$, Fig. 20a, were also shown in the boundary-layer thickness analysis. It was noted that the vortex remnants could be distinctly seen and the boundary-layer thickness was non-uniform across the span. Turning attention to the reversed-flow thickness (seen as the dark blue region near the wall) it can be seen that this configuration was the most uniform across the span of the four presented.

As was discussed looking at the contours of the response surface equation, micro-ramps without injection, Fig. $20 \mathrm{c}$, had little effect on the reversed-flow thickness compared to the baseline value of $0.973 \mathrm{~cm}$ when $s=35 \mathrm{~mm}$. In fact, while the reversed-flow thickness was reduced somewhat in line with the devices (at $\mathrm{z}= \pm 1.75 \mathrm{~cm}$ ), it increased slightly between them. With the addition of $1.0 \%$ injection at $s=35 \mathrm{~mm}$, the span-averaged reversed-flow thickness was substantially reduced. This configuration had another important feature to note: it was the only one of the measured configurations to have eliminated the reversed-flow region in line with the devices. It separated the 
spanwise separation into pockets with regions of attached flow between, which may decrease the unsteadiness due to the separation.

\section{Optimization}

The models that were developed can be used to predict the expected values within the range of the factor variables tested. They can also, then, be used to find values of the factor variables which optimize (for our responses, that is minimize) the response variables. First, a factor-variable optimization was performed based only on the boundary-layer thickness. Next a factor-variable optimization was performed based only on the reversed-flow thickness. And last, the optimization was performed based on the both response variables jointly. Table 6 shows the results for optimization study. All response values are span averaged as described previously. Although, the most significant reduction (compared to the baseline) in both response variables was observed when optimized individually, the results ignored the effect on the other response variable. Thus, a joint optimization of both response variables was necessary which predicted the best possible hybrid flow control configuration within the range of the factor variables tested.

Table 6. Factor-Variable Optimization

\begin{tabular}{|c|c|c|c|c|}
\hline \multirow{2}{*}{ Factor Variables } & \multirow{2}{*}{ Baseline } & \multicolumn{3}{|c|}{ Optimized Variables } \\
\cline { 3 - 5 } & & $\begin{array}{c}\text { Boundary-Layer } \\
\text { Thickness }\end{array}$ & $\begin{array}{c}\text { Reversed-Flow } \\
\text { Thickness }\end{array}$ & Joint \\
\hline \hline $\begin{array}{c}s \\
{[\mathrm{~mm}]}\end{array}$ & 0.0 & 28.93 & 35.00 & 31.14 \\
\hline $\begin{array}{c}h \\
{[\mathrm{~mm}]}\end{array}$ & 0.0 & 5.0 & 5.0 & 5.0 \\
\hline $\begin{array}{c}c^{*} \\
{[\mathrm{~mm}]}\end{array}$ & 0.0 & Not a Factor* & 24.0 & 24.0 \\
\hline $\begin{array}{c}I F R \\
{[\mathrm{~mm}]}\end{array}$ & 0.0 & 1.0 & 1.0 & 1.0 \\
\hline \hline \multicolumn{2}{|c|}{ Predicted Response Values } & $\mathbf{2 . 2 4 8}$ \\
\hline \hline $\begin{array}{c}\delta \\
\mathrm{cm}]\end{array}$ & 2.89 & $\mathbf{2 . 2 2 9}$ & - & $\mathbf{0 . 3 2 4}$ \\
\hline $\begin{array}{c}R F T \\
{[\mathrm{~cm}]}\end{array}$ & 0.973 & - & $\mathbf{0 . 2 7 8}$ & \\
\hline
\end{tabular}

* The factor chord length was not included in the boundary-layer thickness response equation, see Equation 1. Therefore, predictions of boundary layer thickness are independent of chord length.

\section{Corner Profiles}

Recent studies ${ }^{10}$ have shown that applying flow control to the centerline of rectangular cross section facilities can cause unwanted effects in the corners in the form of corner vortices. Further, the greater the improvement on the centerline flow, the greater seems to be the deterioration of the flow in the corners.

Normalized total pressure profiles measured along a 45-degree angle in the corner are presented in Fig. 21. All of the hybrid flow control cases presented in this section have $h=5 \mathrm{~mm}$ and $c=24 \mathrm{~mm}$, so they will be distinguished by spacing and injection flow ratio in the discussion. The curves fall roughly into two groups. The first includes the baseline, injection only, and $s=35 \mathrm{~mm}$, IFR $=0.0 \%$ configurations, which have reversed flow thicknesses of $0.973,0.903$, and $0.845 \mathrm{~cm}$ respectively. The second group includes the configurations with $s=25$ $\mathrm{mm}, I F R=0.0 \% ; s=25 \mathrm{~mm}, I F R=1.0 \%$; and $s=35 \mathrm{~mm}, I F R=1.0 \%$. These configurations have reversed-flow thicknesses of $0.338,0.389$, and $0.275 \mathrm{~cm}$ respectively. While not enough data are available to draw definitive conclusions, the available data seem to agree with what is expected based on the prior studies. 


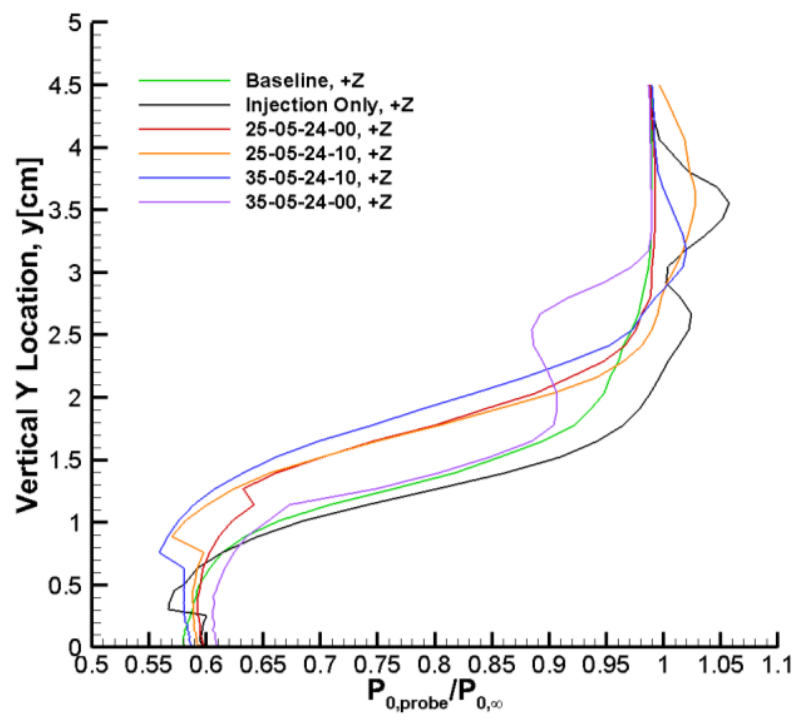

Figure 21. Normalized total pressure profiles in the corner, measured at a 45-degree angle. The form of the line labels is $s-h-c-(10 * I F R)$.

\section{Conclusions}

Hybrid flow control was able to reduce the boundary-layer thickness and reversed-flow thickness caused by a normal shock boundary layer interaction. Compared to the baseline uncontrolled case which had a boundary-layer thickness of $2.89 \mathrm{~cm}$ and a reversed-flow thickness of $0.973 \mathrm{~cm}$, hybrid flow control configurations generated spanaveraged boundary-layer thicknesses as low as $2.15 \mathrm{~cm}$ and reversed-flow thicknesses as low as $0.207 \mathrm{~cm}$. Improvements were made with micro-ramps only or in the hybrid configuration. Large micro-ramps $(h=5 \mathrm{~mm}, c=$ $24 \mathrm{~mm}$ ) closely spaced caused the most two-dimensional separation region. Large ramps widely spaced with $1.0 \%$ injection flow ratio was the only configuration to eliminate the separation in line with the devices and break the separation into pockets. Injection was able to make the boundary layer thickness more uniform by reenergizing the velocity deficit region created by the ramps.

Response surface equations were obtained for the response variables in terms of the factor variables tested. The boundary-layer thickness could be modeled with as little as two variables and their interaction, however a more complete model provided slightly better results. Spacing was chosen as a factor in the equation rather than chord length because while both provided statistically valid models, spacing could be explained physically.

The reversed-flow thickness required many terms including higher order interactions to get a statistically significant model. A one factor at a time analysis would have missed the interactions that were necessary to understand the effects of the hybrid flow control.

The hybrid flow control was optimized for this tunnel configuration (e.g. with a parabolic bump to create a normal shock and incoming Mach number of 0.67) on the basis of the response surface equations obtained. A joint optimization of boundary layer thickness and reversed-flow thickness showed the optimum configuration to be $s=31.14 \mathrm{~mm}, h=5 \mathrm{~mm}, c=24 \mathrm{~mm}$, and $I F R=1.0 \%$. For these factor values, the predicted boundary layer thickness was $2.248 \mathrm{~cm}$, and the predicted reversed-flow thickness was $0.324 \mathrm{~cm}$. The value of spacing optimized within the factor range while height, chord length, and injection flow ratio optimized at their maximum values. The maximum levels of these factors were selected based on the screening study. Additionally considering that increasing the height or chord length of the devices increases drag and the injection flow has to come from elsewhere in the propulsion system, it would be necessary to consider additional response variables to determine if these factors could be increased further.

It was noted that the corner flow was influenced by attempts to control the flow on the centerline of the tunnel.

\section{References}

${ }^{1}$ Vyas, M.A., Hirt, S.M., Anderson, B.H., "Experimental Investigation of Normal Shock Boundary-Layer Interaction with Hybrid Flow Control," AIAA Paper 2012-48, 2012.

${ }^{2}$ Anderson, B. H., Tinapple, J., Surber, L., "Optimal Control of Shock Wave Turbulent Boundary Layer Interactions using Micro-Array Actuation,” AIAA Paper 2006-3197, 2006. 
${ }^{3}$ Hirt, S. M., Anderson, B. H., "Experimental Investigation of the Application of Microramp Flow Control to an Oblique Shock Interaction," AIAA-2009-919, 2009.

${ }^{4}$ Babinsky, H., Li, Y., Pitt Ford C. W., "Microramp Control of Supersonic Oblique Shock-Wave/Boundary-Layer Interactions," AIAA Journal, Vol. 47, No. 3, 2009.

${ }^{5}$ Herges, T., Kroeker, E., Elliott, G., Dutton, C., "Micro-Ramp Flow Control of Normal Shock/Boundary-Layer Interactions," AIAA Paper 2009-920, 2009.

${ }^{6}$ Galbraith, M. C., Orkwis, P. D., "Multi-Row Micro-Ramp Actuators for Shock Wave Boundary-Layer Interaction Control," AIAA Paper 2009-321, 2009.

${ }^{7}$ Lee, S., Goettke, M. K., Loth, E., Tinapple, J., Benek, J., “Microramps Upstream of an Oblique-Shock/Boundary-Layer Interaction," AIAA Journal, Vol. 48, No. 1, 2010.

${ }^{8}$ Lakebrink, M.T., Mani, M., Mace, J.L, McMillan, M.L., "Numerical Investigation of Fluidic Hybrid Flow Control Influence on Boundary Layer Characteristics," AIAA Paper 2009-4014, 2009.

${ }^{9}$ Hirt, S.M., Zaman, K. B. M. Q., Bencic, T. J., "Experimental Study of Boundary Layer Flow Control Using an Array of Ramp-Shaped Vortex Generators,” NASA/TM-2012-217616, AIAA Paper 2012-741, 2012.

${ }^{10}$ Burton, D., Babinsky, H., Bruce, P., "Experimental investigation into Parameters Governing Corner Interactions for Transonic Shock-Wave/Boundary-Layer Interactions,” AIAA Paper 2010-871. 